\title{
Performance Improvement of the Tracking System of a Satellite Laser Communication
}

\author{
Nourin Kadir, Ariq I. Aziz, Sarjana J. Chowdhury, Samiha Shamma and Mohammed \\ Tarique \\ Department of Electrical \& Electronic Engineering, American International University- \\ Bangladesh, Banani, Dhaka - 1213
}

\begin{abstract}
Free Space Optics (FSO) is an optical communication technology that uses light propagating in free space to transmit data between two points. The technology is useful where the physical connections by the means of optical fibre cables are impractical to use due to high costs or other considerations. FSO has been proposed by using a laser. FSO is considered as one of the most popular choices for inter-satellite communication where the performance is limited by "satellite vibration". FSO cannot achieve a stable and 'healthy' link due to such type of vibration. The ultimate disadvantages include misalignment in tracking, signal attenuation and high BER (bit error rate). Hence the satellite vibrations need to be compensated (or minimized). This paper introduces the feed-forward vibration compensation model by introducing PID controller. The simulation results indicate that the proposed method minimizes the vibration effects and hence reduces BER in satellite laser communication system [7].
\end{abstract}

\section{General Terms}

Satellite, Vibration effect, PID controller, Feed-forward compensator.

\section{Keywords}

FSO, satellite vibration, tracking system, BER, compensation model.

\section{INTRODUCTION}

Presently approximately 2,000 artificial satellites orbiting the earth, provide an continuous communication between any places on earth. The most important issue for satellite communication is the tracking between two satellites [8]. But this fine tracking is greatly affected by vibration due to continuous movement of the satellites. Some of the problems related to the satellite vibrations include (a) deviation from the line of sight, which affects the fine tracking system, (b) misalignment between the transmitter scheme and the receiver scheme, (c) signal attenuation, and (d) High BER [1].

Several solutions have been proposed in the literatures for compensating the vibration effects (a) bandwidth adaptation, (b) beamwidth adaptation, (c) Power Control, (d) coding technique, (e) channel diversity, (f) vibration isolator, and (g) self-tuning feed-forward compensation. In this paper we focus on the selftuning feed-forward compensation technique. This paper provides an approach to develop a feed-forward compensation model [2] by introducing PID controller in the fine tracking system to minimize the vibration effect. MATLAB/Simulink has been used as a simulation tool. A mathematical model has also been proposed in this paper for the BER as a function of vibration amplitude.

\section{LINK DESIGN}

In case of FSO communication the optical communication is used as a carrier that enables the transmission scheme to use a very narrow beam divergence (micro radians). To ensure correct reception of this very narrow beam the receiver needs to be perfectly aligned with the transmitter [11-12]. But the transmitted beam may miss the pair satellite sometimes because of vibration effects. This phenomenon creates delay in further proliferation in FSO communication [9-10]. For the statistical description of pointing errors [3] the Rayleigh distribution could be used. As it is indicated by the Rayleigh model that the azimuth and elevation tracking processes are independent and identically distributed, so that the radial pointing error [13-16] may be expressed by the following pdf (probability density function) [4]-

$f(\theta)=\frac{\theta}{\sigma^{2}} e^{-\frac{\theta^{2}}{2 \sigma^{2}}}$

Where, $\theta$ is the radial pointing error and $\sigma$ is the radial pointing error standard deviation (vibration amplitude). So the link equation can be expressed as [5-6]

$P_{r}(\theta)=P_{t} G_{t} \eta_{t} L_{r} G_{r} \eta_{r} e^{\left(-G_{t} \theta^{2}\right)}$

Where,

$P_{r}(\theta)$ is the power of the received signal

$P_{t}$ is the power of the transmitted signal

$G_{t}$ is the gain of transmitter antenna

$G_{r}$ is the gain of the receiver antenna

$\eta_{t}$ is the transmitter efficiency

$\eta_{r}$ is the receiver efficiency

$L_{r}$ is the free space range loss

and $e^{\left(-G_{t} \theta^{2}\right)}$ is the pointing loss factor.

\section{BER and VIBRATION AMPLITUDE}

BER is widely used to measure the performance of communication system. It is the ratio of number of bits detected with error to the total number of bits transmitted. Here ON/OFF keying is adopted for this link. The receiver has an optical detector which converts the optical power to electronic signal with $\mathrm{R}$ conversion ratio. The receiver's task is to integrate the signal and determine whether the signal is ON or OFF. Before decision making the electronic signal is considered as $y$. Let us assume that noise is independent of the received signal with zero 
mean and variance $\sigma^{2}$. So, when the bit is ON or OFF the signal $y$ is related to the following conditional densities-

$P\left(\frac{y}{o n}, \theta\right)=\frac{1}{\sqrt{2 \pi \sigma_{n}}} e^{\left\{-\frac{\mid y-R P t\left(\left.\theta_{j}\right|^{2}\right.}{2 \sigma_{n}^{2}}\right\}}$

$P\left(\frac{y}{\text { off }}\right)=\frac{1}{\sqrt{2 \pi \sigma_{n}}} e^{\left\{-\frac{|y|^{2}}{2 \sigma_{n}^{2}}\right\}}$

The likelihood function is given by-

$\square(y, \theta)=\frac{P\left(\frac{y}{o n^{\prime}}, \theta\right)}{P\left(\frac{y}{o f f}\right)}=e^{\left\{-\frac{-2 y R P_{r}(\theta)+\left[R P_{r}\left[\theta_{j}\right]\right]^{2}}{2 \sigma_{n}^{2}}\right\}}$ (5)

Taking natural logarithm of both sides of equation (5), cancelling the common factors the following expression is got-

$L \square(y, \theta)=y-\frac{R P_{r}(\theta)}{2}$

........(6)

The BER equation becomes-

$B E R=\int_{0}^{\infty}\left[P(\right.$ on $) P\left(\frac{\text { off }}{\text { on }}, \theta\right)+P($ off $) P\left(\frac{\text { on }}{\text { off }}, \theta\right) \mid f(\theta) d \theta$

where $\mathrm{P}($ off/ on, $\theta)$ and $\mathrm{P}($ on/ off, $\theta)$ are given by-

$P\left(\frac{\text { off }}{\text { on }}, \theta\right)=\int \mathrm{P}_{L \square(y, \theta)<0}\left(\frac{y}{\text { on }}, \theta\right) d y$

$P\left(\frac{\text { on }}{\text { off }}, \theta\right)=\int \mathrm{P}_{L \square(y, \theta)>0}\left(\frac{y}{\text { off }}\right) d y$

.........(9)

Calculating equations (8) and (9) with equations (3) and (4) and then substituting equations (8) and (9) into equation (7), with equation (2) the expression becomes-

$B E R=\int_{0}^{\infty}\left|\left\{\frac{1}{2}-\frac{1}{4} \operatorname{erf}\left(C e^{\left(-G_{t} \theta^{2}\right)}\right)\right\} \frac{\theta}{\sigma^{2}} e^{\left(-\frac{\theta^{2}}{2 \sigma^{2}}\right)}\right| d \theta$

Where $\mathrm{P}($ on $)=\mathrm{P}($ off $)=1 / 2, \mathrm{C}$ is a constant factor, and erf $(\mathrm{x})$ is error function. Here, defining a new variable $U=\theta^{2} / 2 \sigma^{2}$, so the equation number (10) is now changed into the following form-

$B E R=\int_{0}^{\infty}\left[\left\{\frac{1}{2}-\frac{1}{4} \operatorname{erf}\left(C e^{\left.i-2 G_{t} s^{2} u\right)}\right)\right\} e^{(-u)} \mid d u\right.$

Where the vibration amplitude is represented by $G_{t} \theta^{2}$. The following Figure 1 shows the relationship between the satellite vibration amplitude and the BER and it clearly explains that if the vibration amplitude is some how increased then it also increases the BER. It is very obvious that if the BER becomes too large while transmitting then the probability of signal loss will be high too, which is not desirable at all during long distant transmission like data transmission to satellites in space.

The prime objective of this paper is to find out the procedure of minimizing the vibration amplitude of the satellites in space and as well as ensuring fine tracking.

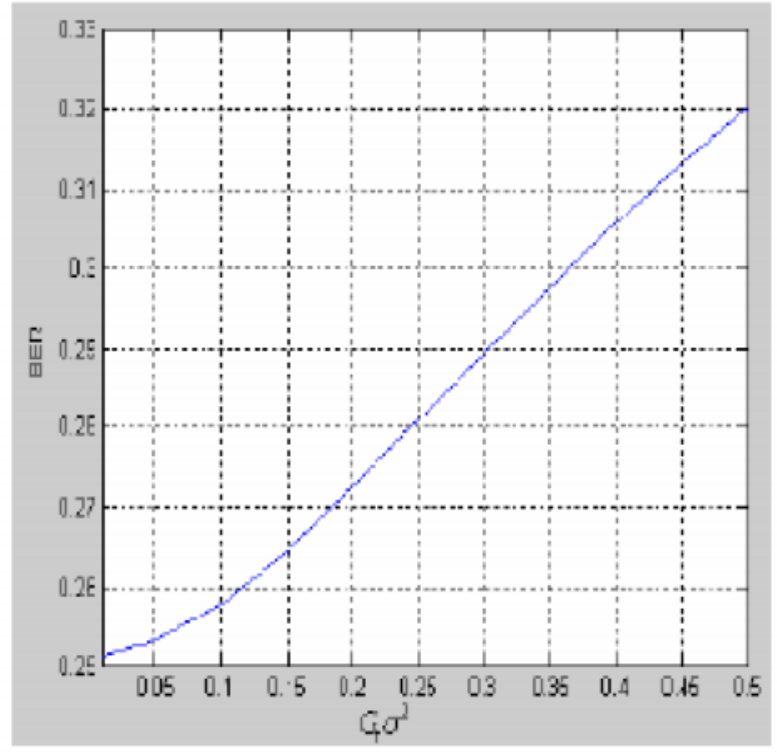

Figure 1: BER as a function of the transmitter gain multiplied by $\sigma^{2}$

\section{FEED-FORWARD VIBRATION COMPENSATION MODEL}

Figure 1 illustrates the generalized relation between the BER and the satellite vibration amplitude. This also implies that the amplitude has a great effect on the system performance. The following section represents a feed-forward vibration compensation model that is developed under fine tracking system using MATLAB/Simulink tool that decreases the vibration effect.

This compensation model in FSO communication is the process where disturbance is added to the output, the output is feedback into the input that can counteract the vibration effect precisely. In our case we add the random number to generate disturbance in the system as practical cases and this disturbance is added up with the original transmitted signal in our system model for compensation. Our prime objective is to find out the best path for fine tracking and here as the transmission medium laser beam is used as we know that laser medium provides very high bandwidth for data transmission. So it is more secure way to use the laser medium rather than the conventional procedure for transmission.

In the feed-forward compensation model the transport delay block is used to hold the transmitted signal for a several seconds, it acts like a buffer here and the zero order hold block is used to digitized the electronic signal for making it suitable to transmit. The following Figure 2 is the schematic representation of our system- 
2:

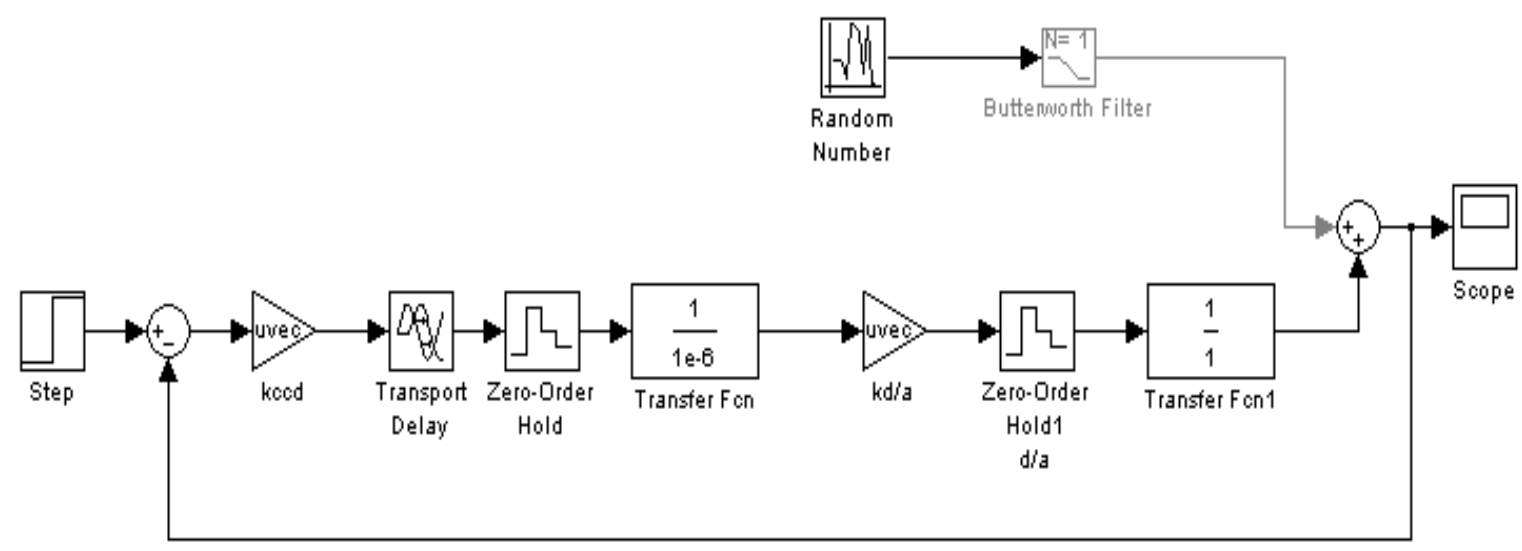

Figure

\section{Circuit diagram with variable transfer function and fixed gain}

This fine tracking system includes step input, transport delay, zero order hold, gain block, random number as noise and butter worth filter.

Figure 2 and Figure 3 show the simulation results before and after compensation respectively. The $\mathrm{X}$ axis represents the sampling time and the $\mathrm{Y}$ axis represents the vibration amplitude. For non compensation model the vibration is almost 0.3 in the following Figure 2. Figure 3 shows that for compensation model the vibration amplitude is minimized to 0.016 . These tow figures are given bellow showing the impact of compensation model-

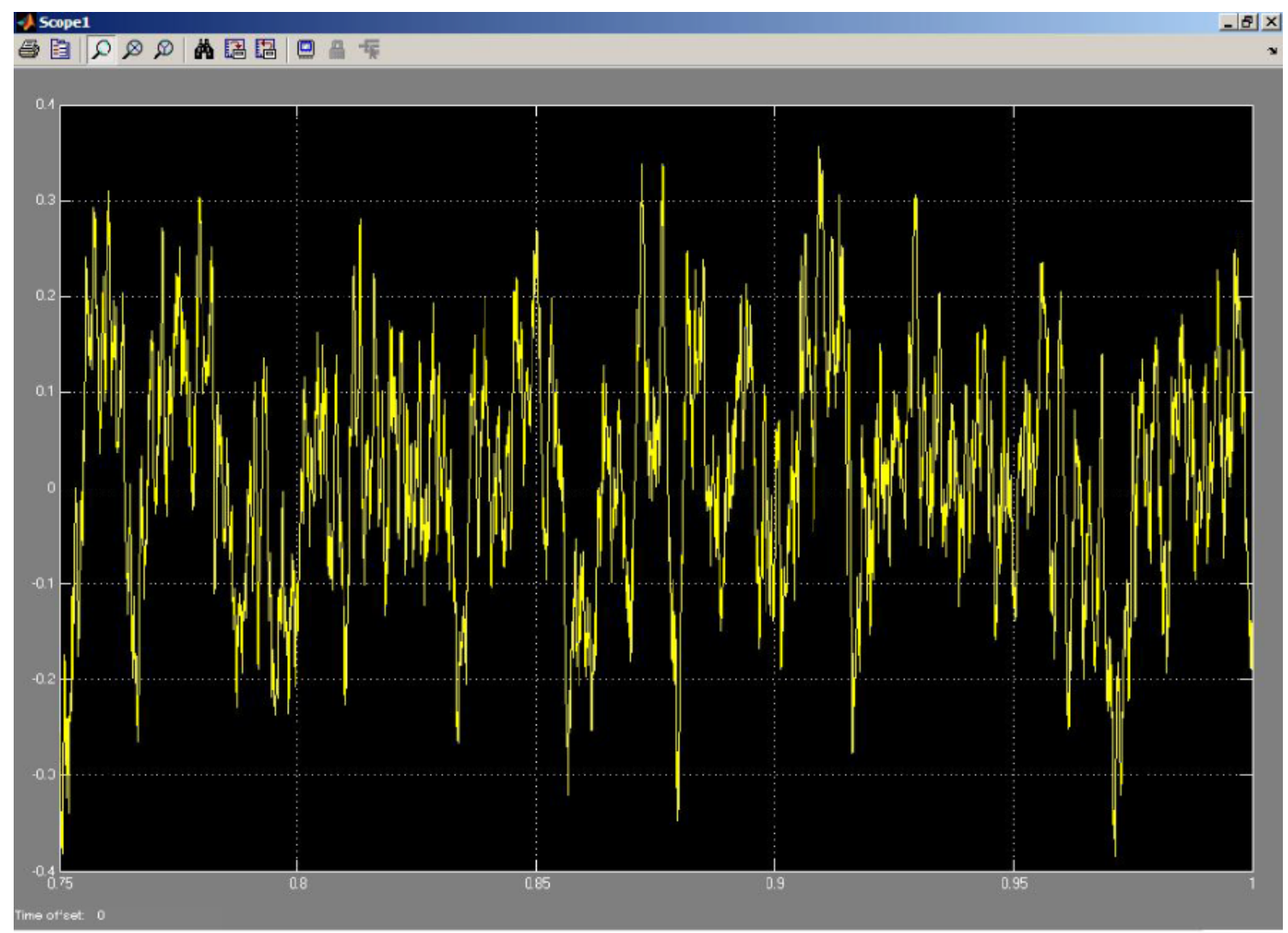

Figure 3: non compensation model 


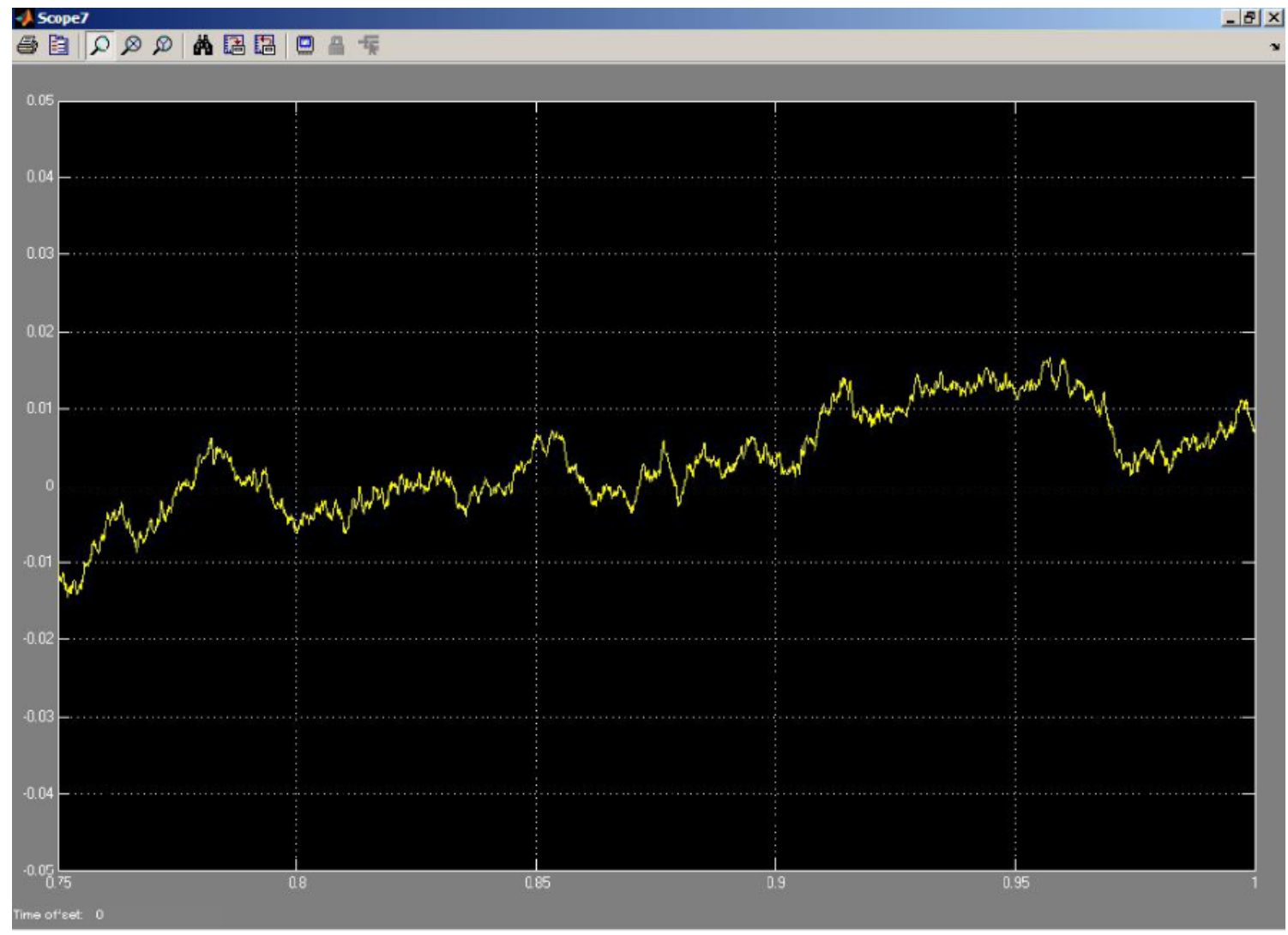

Figure 4: Output of feed-forward compensation model

\section{PID CONTROLLER'S INTRODUCTION}

If PID controller is used in this model then a noticeable change can be observed in the system performance listed in the following table-

Table 1: Performance of PID Controller

\begin{tabular}{|l|l|l|l|l|}
\hline Response & $\begin{array}{l}\text { Rise } \\
\text { time }\end{array}$ & Overshoot & $\begin{array}{l}\text { Settling } \\
\text { time }\end{array}$ & $\begin{array}{l}\text { S-S } \\
\text { error }\end{array}$ \\
\hline $\mathrm{K}_{\mathrm{p}}$ & Decrease & Increase & $\mathrm{NT}$ & Decrease \\
\hline $\mathrm{K}_{\mathrm{i}}$ & Decrease & Increase & Increase & Eliminate \\
\hline $\mathrm{K}_{\mathrm{d}}$ & $\mathrm{NT}$ & Decrease & Decrease & $\mathrm{NT}$ \\
\hline
\end{tabular}

NT: No definite trend.

In words we can define the above four terms such as-

Rise time: the time it takes for the plant output to rise beyond $90 \%$ of the desired level for the first time.

Overshoot: how much the peak level is higher than the steady state, normalized against the steady state condition.

Settling time: the time it takes for the system to converge to its steady state.
Steady-state error: the difference between the steady state output and the desired output.

These four major characteristics are controlled by PID controller. In this segment when PID controller is used instead of conventional gain block for the system, it becomes easy to observe the changes of vibration amplitude by changing $K_{p}, K_{i}$, and $\mathrm{K}_{\mathrm{d} \text {. }}$ A rate limiter block is brought here with PID for limiting first derivative of the signal passing through it.

PID stands for Proportional-Integral-Derivative, referring to the three terms operating on the error signal to produce a control signal. The desired closed loop dynamics is obtained by adjusting the three parameters $\mathrm{K}_{\mathrm{p}}, \mathrm{K}_{\mathrm{i}}$ and $\mathrm{K}_{\mathrm{d}}$, often iteratively by "tuning" and without specific knowledge of a plant model. The effects of these parameters on the system performance have been listed in Table 1. Stability can often be ensured using only the proportional term. The integral term permits the rejection of a step disturbance (often a striking specification in process control). The derivative term is used to provide damping or shaping of the response.

Where the equation for PID controller is-

$U(s)=\left(K_{p}+K_{i} \frac{1}{s}+K_{d} s\right) e(s)$

With PID controller transfer function-

$C(s)=\left(K_{p}+K_{i} \frac{1}{s}+K_{d} s\right)$ 


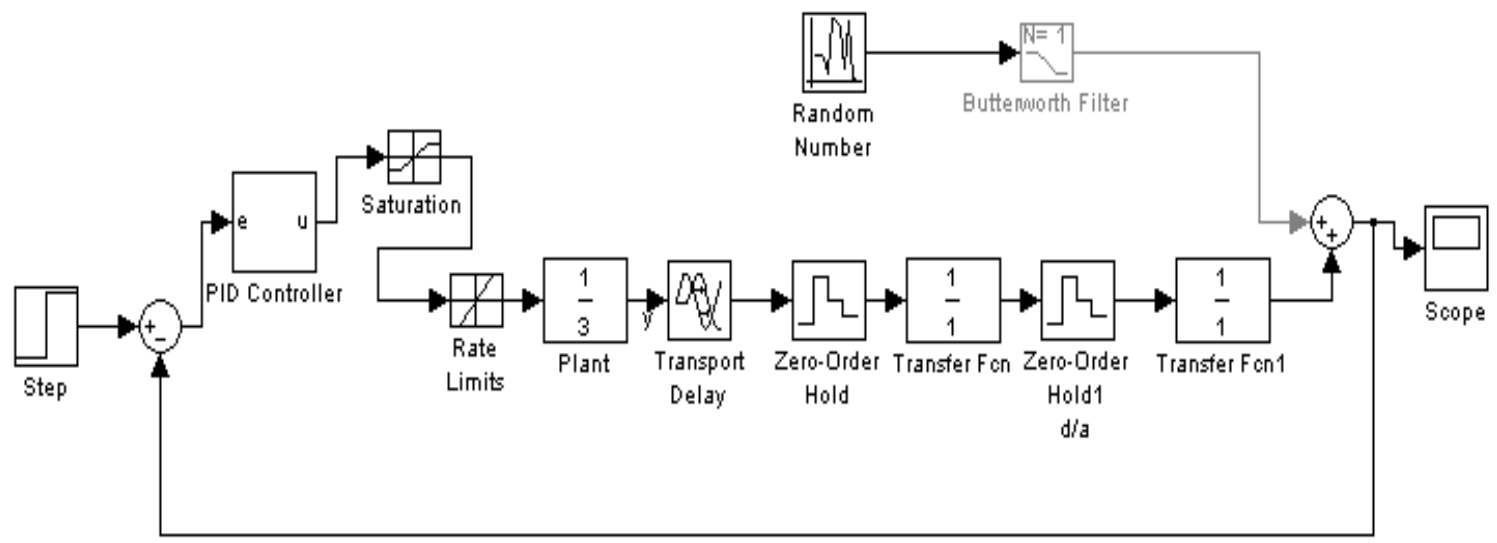

Figure 5: system model with PID controller

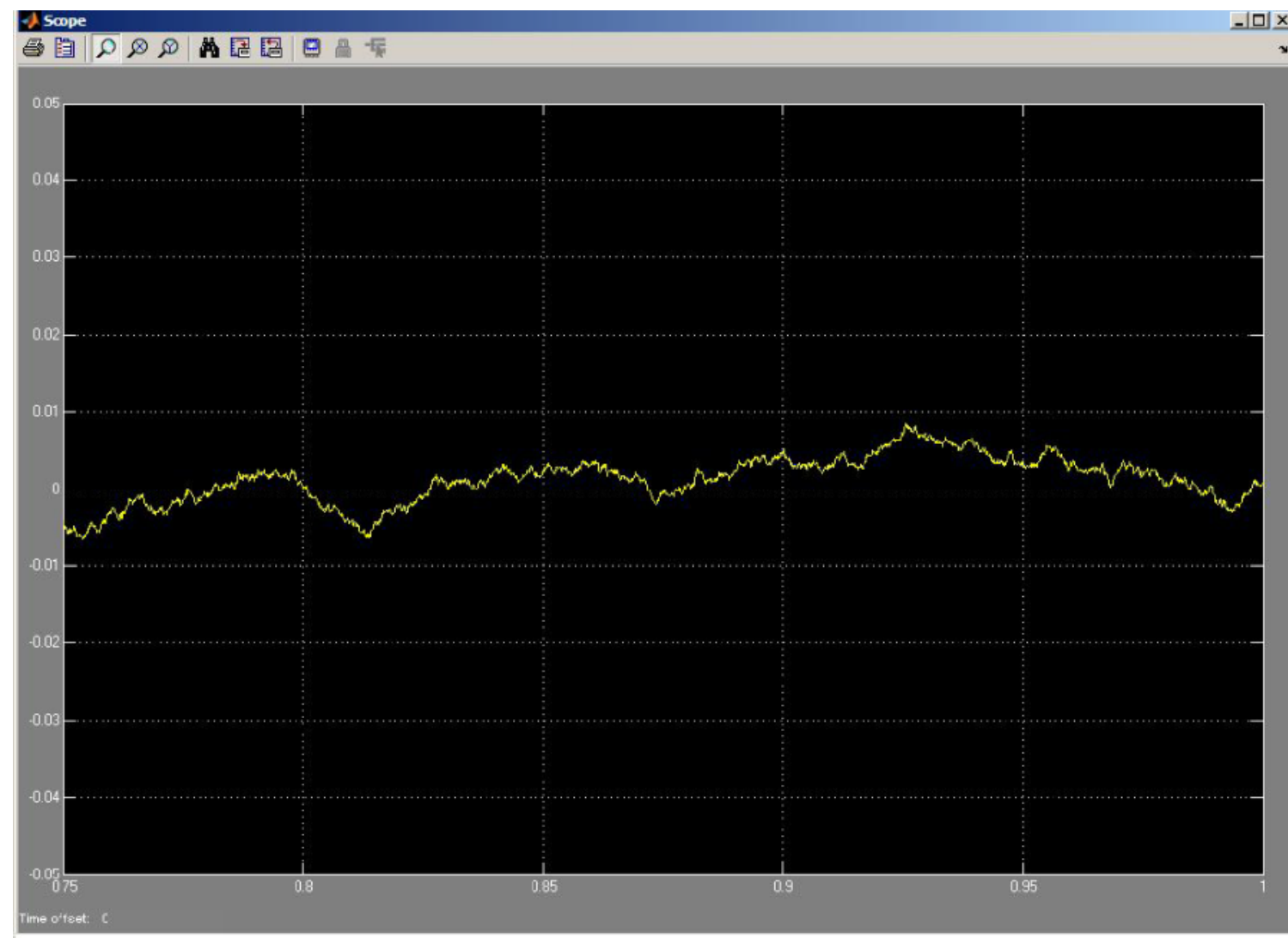

Figure 6: simulation using PID controller

Figure 5 shows the block diagram of the fine tuning system model where PID controller is introduced and Figure 6 indicates the improved result for introducing PID controller.

The overall vibration amplitude of the satellite is reduced and consequently the BER is also minimized noticeably giving higher probability of precise line of sight (LOS) between satellites in case of fine tracking.
The following table shows the comparison between the system model with PID controller and the system model without PID controller.

From the following table it can be clearly seen that the performance of the tracking is far better in case of using the PID controller. Here data is presented for time interval of 0.5 second covering the span from 0.75 to 1.00 second- 
Table 2: comparison between model with PID controller and without PID controller

\begin{tabular}{|l|l|l|}
\hline Time & $\begin{array}{l}\text { Noise with PID } \\
\text { controller (the Y } \\
\text { axis in the graph) }\end{array}$ & $\begin{array}{l}\text { Output with } \\
\text { function and transfer } \\
\text { fixed gain (the Y } \\
\text { axis in the } \\
\text { graph) }\end{array}$ \\
\hline 0.75 & -0.005 & -0.012 \\
\hline 0.8 & 0.00 & -0.005 \\
\hline 0.85 & 0.0025 & 0.008 \\
\hline 0.9 & 0.004 & 0.004 \\
\hline 0.95 & 0.003 & 0.012 \\
\hline 1.00 & 0.001 & 0.008 \\
\hline
\end{tabular}

Now from this table it is depicted that the PID controller gives better performance than the conventional feed-forward compensation model as shown in Figure 6.

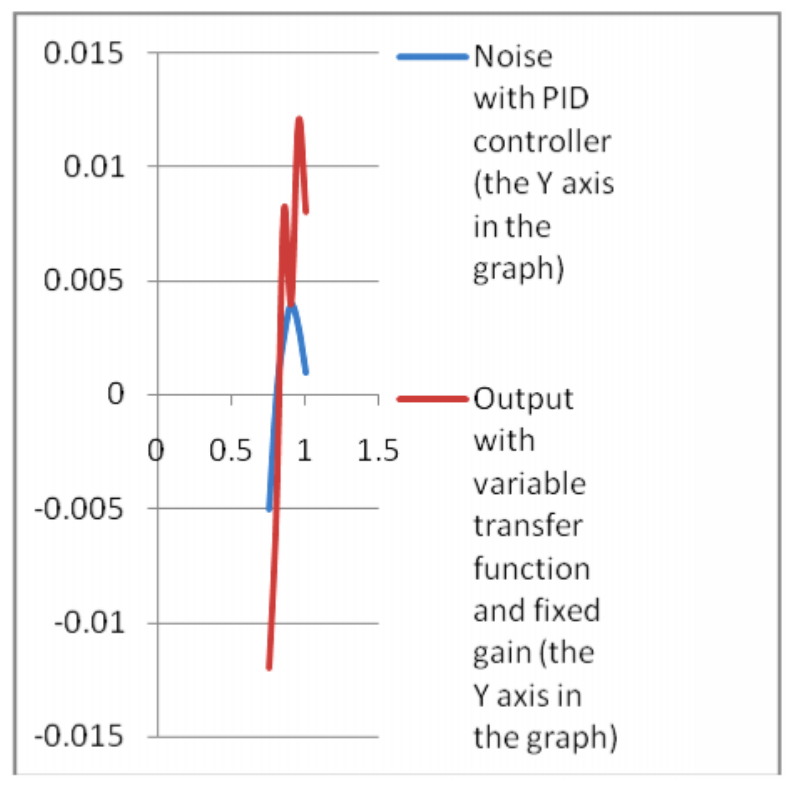

Figure 6: Result comparison graph

\section{CONCLUSION}

The above analysis clearly explains that if the PID controller is used instead of conventional feed-forward compensation model the BER is further reduced and the fine tuning system becomes more precise. The paper discusses the vibration effect on BER and the minimization of system BER. First a Rayleigh density function is figured out for pointing error and later on the model is developed for minimizing BER against vibration amplitude as the vibration amplitude has tremendous effect on BER. Concept of using PID controller is brought freshly and the result is better than using feed-forward compensator for fine tuning model.
Using PID controller the vibration amplitude decreases up to 0.004 at sampling time 0.9 second.

\section{REFRENCES}

[1] WANG Jian-min, TANG Jun-xiong, SUN Dong-Xi, ZHU Yan \& GU Ya-quan, " Study on the Uniform Beacon Used for the Free Space Laser Communication. Acta OpticaSinica, January, 2006, 26(1).

[2] MA Jing, HAN Qi-qi, YU Si-yuan, TAN Li-ying and GUAN Wen-cheng, "The Effect of Vibration on Intersatellite Optical Communication and the Resolving" Project. Laser Technology, June, 2005, 29(3).

[3] Shlomi Arnon and N.S. Kopeika," Laser Satellite

Communication Network-vibration Effect and Possible Solutions", Proceedings of the IEEE, October, 1997, 85(10): 1646-1661.

[4] Morio Toyoshima, Takashi Jono, Keizo Nakagawa and Akio Yamamoto, "Optimum Divergence Angle of a Gaussian Beam Wave in the Presence of Random Jitter in Free-space Laser Communication Systems", Journal of Optics Society of America, 2002, 19 (3): 567-571.

[5] Stephen G. Lambert \& William L. Casey," Laser

Communications in Space. Artech. House, Inc., Norwood, Massachusetts, 1995: 95.

[6] Shlomi Arnon and Kopeika Norman S. "Performance Limitation of Free-space Optical Communication Satellite Network due to Vibrations-analog Case", Optical Engineering, 1997, 36(1): 175-182.

[7] B. I. Edelson and G. Hyde, "Laser satellite communications program technology and applications," IEEE-USA Aerospac Policy Committee Rep., Apr. 1996.

[8] D. K. Paul, F. Faris, R. Garlow, T. Inukai, B. Pontano, R. Razdan, A. Ganz, and L. Caudill, "Optical intersatellite links: Application to commercial satellite communications," in Proc. 14th AIAA Int. Communication Satellite Systems, Washington, D.C., Mar. 22-26, 1992, pp. 277-289.

[9] D. K. Paul, "Optical cross links for advanced Satcom networks,"presented at the Asia Pacific Microwave Conf., New Delhi, India, Dec. 17-20, 1996.

[10] M. Fujise, M. Nohara, K. Uehara, and W. Chujo, "Broadband mobile satellite communication system by LEO-SAT and optical ISL's," in Proc. IEEE GLOBECOM, London, vol. 1, 1992, pp. 437-442.

[11] M. Wittig, L. van Holtz, D. E. L. Tunbridge, and H. C. Vermeulen, "In orbit measurements of micro accelerations of ESA's communication satellite OLYMPUS," in Selected Papers on Free-Space Laser Communication II-SPIE Ms 100, D. L. Begly and B. J. Thompson, Eds. Bellingham, WA: SPIE,1994, pp. 389-398.

[12] S. Dyne, P. P. Collins, and D. Tunbridge, "Satellite mechanical health monitoring," in IEE Colloquium Advanced Vibration Measurements, Techniques and 
Instrumentation for the Early Predication of Failure, 1992, pp. $4 / 1-4 / 8$.

[13] S. J. C. Dyne, D. E. L. Tunbridge, and P. P. Collins, "The vibration environment on a satellite in orbit," in IEE Colloquium High Accuracy Platform Control in Space, 1993, pp. 12/1-12/6.

[14] K. J. Held and J. D. Barry, "Precision pointing and tracking between satellite-borne optical systems," Opt. Eng., vol. 27,no. 4, pp. 325-333, Apr. 1988.

[15] C. C. Chen and C. S. Gardner, "Impact of random pointing and tracking errors on the design of coherent and incoherent optical intersatellite communication links," IEEE Trans. Commune., vol. 37, pp. 252-260, Mar. 1989.

[16] J. D. Barry and G. S. Mecherle, "Beam pointing error as a signify- cant parameter for satellite borne, free-space optical communication systems," Opt. Eng., vol. 24, no. 6, pp. 1049-1054, December, 1985. 